Jianfeng Xiong, Youhua $\mathrm{Xu}^{*}$ and Quan Zhu

State Key Laboratory of Quality Research in Chinese Medicine, Faculty of Chinese Medicine, Macau University of Science and Technology, Avenida Wai Long, Taipa, Macau

Dates: Received: 13 October, 2014; Accepted: 21 November, 2014; Published: 24 November, 2014

*Corresponding author: Youhua Xu, M.D., Ph.D. State Key Laboratory of Quality Research in Chinese Medicine, Faculty of Chinese Medicine, Macau University of Science and Technology, Avenida Wai Long, Taipa, Macau. Tel: +853-88972452; Fax: +85328825123; E-mail: yhxu@must.edu.mo

www.peertechz.com

ISSN: 2455-8583

Keywords: Diabètes mellitus ; Inflammation ; Oxidative stress; Radix astragali

\author{
Review Article
}

\section{Anti-Inflammation effects of Radix Astragali Plays an Important Role in Ameliorating Type 2 Diabetes Mellitus}

\section{Introduction}

Diabetes mellitus (DM), namely Xiao Ke in traditional Chinese Medicine, is a group of metabolic diseases in which a person appears over-high concentration of blood glucose. With the increment in the number of elderly population and the prevalence of obesity, the World Health Organization (WHO) estimates that more than 347 million people worldwide have diabetes and diabetes deaths will double between 2005 and 2030 without urgent action [1]. In China, the Chinese Diabetes Society estimates that 92.4 million people have DM, and more than $90 \% \mathrm{DM}$ cases are suffering from type 2 diabetes (T2DM) [2]. DM often accompanies with a series of complications including cardiovascular and neuropathic diseases and seriously decreased the quality of life in DM population [2]. Therefore, preventing and controlling the progression of DM is of great significance.

DM mainly includes 2 types, namely type 1 (T1DM) and T2DM. T1DM results from the body's failure to produce enough insulin, thus daily administration of insulin is the main solution. Distinguished from T1DM, T2DM, as the major form of DM, is characterized by high plasma level of insulin accompanied with insulin resistance in peripheral tissues [3]. However, with the lasting of the disease, the beta cells from is let will occur damage or even death, which will result in insulin deficiency. In that case, insulin should be administrated to the patients. Typical drug treatments for T2DM mainly include insulin sensitizers, e.g. rosiglitazone [2,4], sulfonylurea [2] and metformin [2,5]. Al though effective at reducing the process of T2DM, these drugs may cause serious side effects. It has documented that thiazolidinediones (TZDs), a group of insulin sensitizer medicines which are widely used in clinic, could increase risk ofheart failure and bone fractures [6,7]. Previously, it was reported that pioglitazone and rosiglitazone showed modest or even negative effects on blood lipid variables in DM patients [8]. Fibrates, Peroxisome proliferatoractivated receptor alpha (PPAR- $\alpha$ )-agonist, which are effective in lowering triglycerides and low-density lipoprotein (LDL) while raising high-density lipoprotein (HDL) levels in dyslipidemic patients, were found to have no sufficient activity as anti-diabetic agents [9].

Traditional Chinese Medicines, with multi-target therapeutic effects and low side effects, have been proven to possess availability and effectiveness towards T2DM and its complications clinically. Radix Astragali $(R A)$ is one of the most common used Chinese Medicines in clinic and T2DM has now been widely recognized as a "low grade inflammatory disease", the present article aims to review the therapeutic effects of $R A$ on DM, especially T2DM, from the perspective of inflammation.

\section{Radix astragali and T2DM}

Radix Astragali ( $R A$, the root of Astragalusmembranaceus), which can refuel energy, enhance the immune system, expel the toxin, promote health activities and promote skin growth etc., is a common Chinese herbal medicine. In traditional Chinese Medicine, $R A$ was often applied accompanying with other herbs, such as Angelica and Ginseng, to constitute various complex prescription formulas. Such herbal formulas have been used for thousands of years in China to treat strokes, tumors, kidney dysfunctions etc. [10,11].

The use of $R A$ to treat DM, namely Xiao Ke in Chinese Medicine, has been recorded in Shen Nong's Classic of Materia Medica (Shen Nong Ben Cao Jing) in the Western Han Dynasty (206 BC - 24 AD). Reports from both clinic and experimental studies have observed satisfactory effects of $R A$ on the classical symptoms of T2DM including polyuria, polydipsia and polyphagia [12-14]. Currently, most diabetic herbal formulas sold in the market contain $R A$. However, the exact active components of $R A$ and its diabetic-ameliorating mechanisms have not yet been fully understood. 
It has been demonstrated that inflammatory factors are significant enhancer of insulin resistance. Amounts of inflammatory cytokines could be detected in apparently "healthy" individuals who later develop into T2DM [15-17], suggesting that inflammation occurs early during the period of impaired glucose tolerance and appeared before the diagnosis of T2DM. In fact, recent investigation have demonstrated that pro-inflammatory molecules including inducible nitric oxide (NO), cytokines such as tumor necrosis factor-alpha (TNF- $\alpha$ ), interleukine-6 (IL-6) and advanced glycosylation end products (AGEs) could lead to inflammation and promote the process of insulin resistance [18]. Therefore, T2DM has been recognized as a low grade inflammatory disease $[5,19]$.

\section{Inflammation contributes to insulin resistance in T2DM}

Recently, much has been learned about the intracellular signaling pathways activated by inflammatory and insulin signaling [20]. Insulin affects cells through binding to its receptor on the insulin-responsive cell membrane. The stimulated insulin receptor phosphorylates itself and the substrates (insulin receptor substrate, IRS), and finally initiate downstream signaling events. It has been demonstrated that the inhibition of signaling downstream of the insulin receptor is a primary mechanism through which inflammatory signaling leads to insulin resistance [21]. Amounts of studies have shown that exposure of cells to inflammatory factors, such as TNF- $\alpha$, could inhibit the phosphorylation of IRS $[16,22,23]$ and finally induce insulin resistance. In this sense, ameliorating inflammation possesses potential role in controlling T2DM.

The nuclear factor-kappa B (NF- $\mathrm{B}$ ) pathway has been well known to play an important role in inflammation-induced insulin resistance. Report indicated that inflammatory cytokines could stimulate I-kappa-B (IкB) kinase- $\beta$ (IKK $\beta$ ), which is a downstream inhibitor of insulin receptor, and induce the activation of NF- $\kappa B$ [24]. Following activation, NF- $\mathrm{kB}$ translocates to the nucleus, increases the expression of inflammatory cytokines and finally promotes development of insulin resistance [24]. Therefore, NF- $\kappa$ B plays in a central role in this vicious cycle and the inhibition of NF- $\mathrm{kB}$ activation should help to ameliorate T2DM.

\section{RA directly inhibited diabetic inflammation}

As discussed above, the use of $R A$ to treat DM has received satisfactory effect, e.g. it can alleviate diabetic albuminuria. However, its exact mechanism is not well recognized. Known biologically active fractions of $R A$ mainly include two classes of chemical compounds, flavonoids and saponins [25]. The effect of components from $R A$ on hyperglycemia has been demonstrated. Recently, Hoo and colleagues [18] found the active fraction from $R A$ including calycosin-7$\beta$-D-glucoside, ononin, calycosin and for mononetin etc. could significantly decrease the plasma glucose and triglyceride levels in $\mathrm{db} /$ $\mathrm{db}$ mice via inhibiting inflammatory process [18].

Studies suggested that inflammatory cytokines,TNF- $\alpha$ for example, can inhibits insulin sensitivity by activating different key steps in the insulin signaling pathway [26-28], while active fraction of $R A$ (eg. calycosin) could significantly reduce the secretion of pro-inflammatory cytokines (eg. TNF- $\alpha$, IL-6and MCP-1) [29] in human THP-1 macrophages and lipopolysaccharide (LPS)- induced activation of NF- $\mathrm{kB}$ in mouse RAW-Blue macrophages in a dose-dependent manner. Most recently, Li and colleagues [30] demonstrated that a component from $R A$ namely Astragaloside IV could decrease high-glucose-induced translocation of NF- $\kappa \mathrm{B}$ p65 and increase matrix metalloproteinase (MMP)-2 expression in bone marrow mesenchymal stem cells; more importantly, Astragaloside IV could decrease TNF- $\alpha$ expression in patients and ameliorate high blood glucose. Hoo and colleagues [18] found active components of $R A$ could directly inhibit LPS-induced NF- $\mathrm{kB}$ activity in mouse macrophages. Advanced glycation end products (AGEs), which were the key initiator and promoter of T2DM, have been demonstrated to be a class of NF- $\kappa \mathrm{B}$-involved inflammation activators [31]. Recently, we also found and reported that calycosinand calycosin-7$\beta$-D-glucoside could significantly decrease inflammatory cytokines secretion, inhibit macrophage inhibition and ameliorate AGEsinduced NF- $\kappa \mathrm{B}$ activation in human umbilical vein endothelial cells (HUVECs) [32,33].

Converging lines above indicate the direct inflammationameliorating effects of active fractions from $R A$ should be one of the pivotal mechanisms that contribute to its therapeutic effects on T2DM.

\section{RA in directly inhibited inflammation via ameliorating oxidative stress}

Hyperglycemia promotes formation of reactive oxygen species (ROS), which can interact with both DNA and proteins, cause mitochondrial superoxide overproduction in cells [34], this could induce or exacerbate intracellular oxidative stress (OS) [34], and finally induce cell and tissue damage. Recent studies indicate that OS contributes a lot to the development of T2DM and diabetic complications $[35,36]$. However, the exact role of OS on T2DM has not been fully understood to now.

Free radicals are formed in T2DM by glucose oxidation or nonenzymatic proteinglycation. Abnormal high levels of free radicals and the simultaneous decline of antioxidant substances can lead to damage of cellular organelles and enzymes, increment of lipid peroxidation, and development of insulin resistance. These increased superoxide production and OS would cause increased formation of AGEs, over-high expression of the receptor for AGEs (RAGE) [34], and finally exacerbate diabetic complications. This process seemed to be more important for T2DM. Therefore, targeting OS is an alternative way towards T2DM treatment. In fact, reports have shown that antioxidant therapy could protects against the development of diabetes in rats [37], and experimental evidence obtained from in vitro studies also showed that prevention of ROS generation could defend against the damaging effects of a hyperglycemic milieu on mesangial cell function [38] (Figure 1).

Studies involving the anti-oxidant effect of $R A$ have been abundant. Flavonoids, which are abundant in $R A$ as discussed above [25], have been demonstrated to protect cells from OS damage [39]. It has been reported from us and other research groups that calycosin has protective effect in endothelial cells against hypoxia-induced barrier impairment [40], and calycosin-7-O- $\beta$-D-glucosidehas free radicals scavenging activity [41] and it could significantly ameliorate 


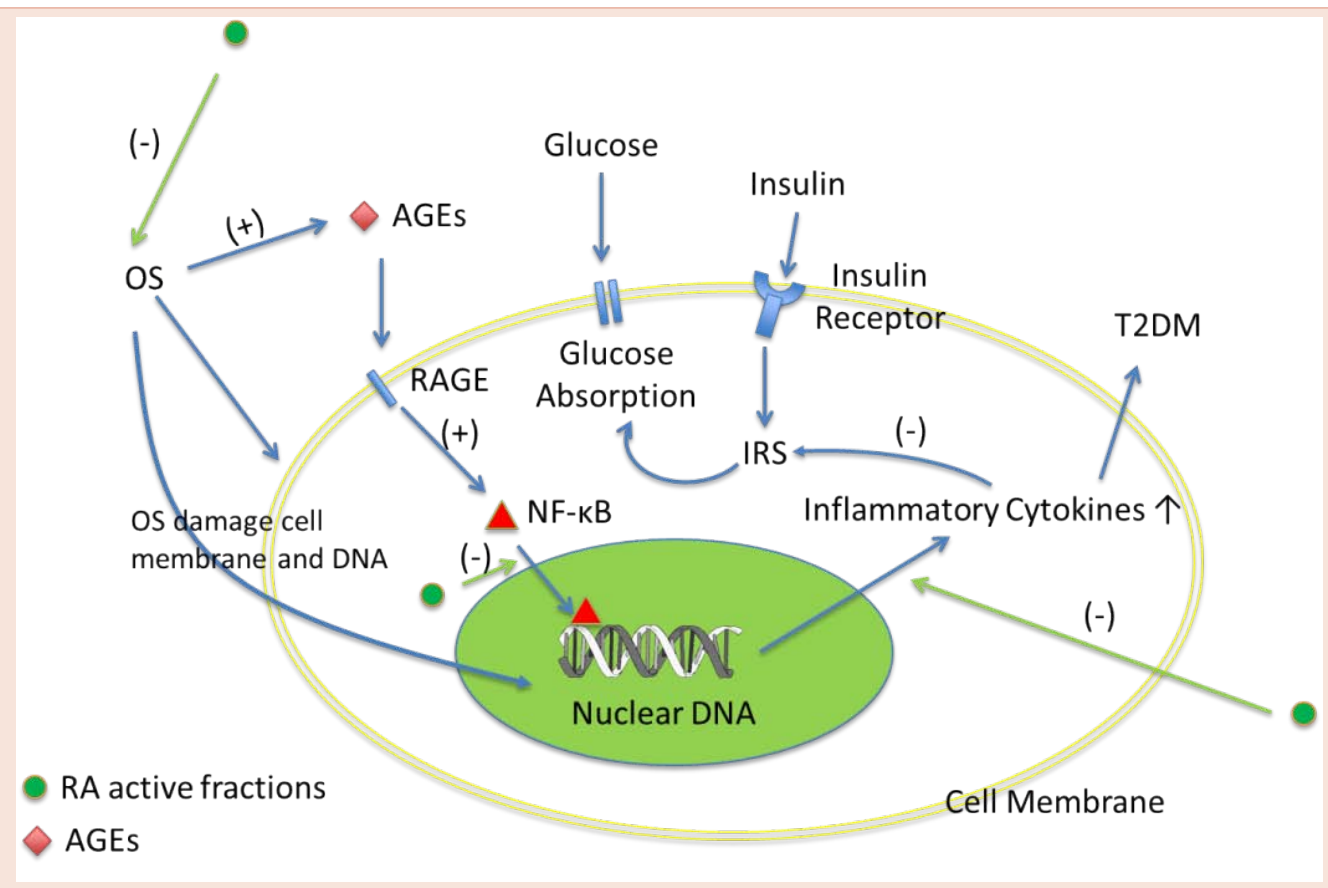

Figure 1: Schematic chart of Radix Astragali's effects against diabetic-inflammation.

AGEs-induced cell OS and apoptosis in a concentration-dependent manner $[32,41]$.

Amounts of reports indicated that besides influences on nitric oxide availability and lipoprotein modifications, the adverse effect of OS on the body inflammatory response may also play a significant role in promoting T2DM $[5,42,43]$. As have been demonstrated, OS will increase the production of oxidative products, AGEs for example, and AGEs have direct effects on inducing diabetic inflammation; thus $R A$ may also function via decreasing AGEs production and finally ameliorating inflammatory signs and symptoms in T2DM (Figure 1).

\section{Others}

Peroxisome proliferator-activated receptor (PPAR)- $\alpha$ is mainly expressed in tissues such as liver, kidney, heart, and muscles where lipoprotein metabolism is important. PPAR- $\alpha$ is the predominant therapeutic target of the fibrates [44]. PPAR- $\gamma$, the target for ligands such as 15-deoxy-prostaglandin J2 and TZDs, is highly expressed in adipose tissue, where it controls insulin sensitivity, adipocyte differentiation, and lipid storage [44]. Pioglitazone and rosiglitazone are efficacious in the treatment of T2DM by control high glucose and prevent a series of critical complications. Summarizing from recent studies, active fraction of $R A$ could active PPAR similarly to that of TZDs $[45,46]$. Formononetin and calycosin have been shown to be activators of PPAR- $\alpha$ and $-\gamma$ [47]. A report from Xu and colleague [48] indicated that astragaloside II and isoastragaloside I from $R A$ have ability to enhance sensitization of insulin thus ameliorate T2DM progression.

Besides activities as discussed above, recent studies have observed some other effects and mechanisms of RA on T2DM. For instance, Xu and colleague [13] indicated that astragaloside II and isoastragaloside I from $R A$ ameliorate glucose intolerance and hyperglycemia by increasing the secretion of the insulin sensitizing hormone and adiponectin from adipocytes. Therefore, converging with the discussions above, $R A$ may ameliorate T2DM via multi-pathways.

\section{Conclusion}

Radix Astragali $(R A)$ has been proven to be availability and effectiveness on T2DM, and recent studies indicate that the active fractions of RA are effective in its complications, such as Diabetic Retinopathy $[48,49]$. Reports suggested that $R A$ possesses multiple activities including anti-oxidant effects and lowering blood glucose; recent studies from us and other research groups indicated that theanti-inflammatory effects of $R A$ may be one of the key mechanisms. Due to its distinct characteristics, e.g. low toxic, multi-target and comprehensive effects etc., its clinical effects are becoming more and more recognized and accepted by modern medicine. However, there are still many works to be done to systemically demonstrate the efficacy and active components of $R A$ on T2DM.

\section{Acknowledgements}

This work was supported by the Science and Technology Development Fund of Macau (FDCT: 049/2012/A2).

\section{References}

1. World Health Organization, Diabetes programme.

2. Chinese Diabetes Society: CHINA GUIDELINE FOR TYPE 2 DIABETES. 2010.

3. Wahren J, Kallas $\AA$ (2012) Loss of Pulsatile Insulin Secretion: A Factor in the Pathogenesis of Type 2 Diabetes? Diabetes 61: 2228-2229. 
4. Sewter C, Vidal-Puig A (2002) PPARy and the thiazolidinediones: molecular basis for a treatment of Syndrome X? Diabetes Obes Metab 4: 239-248.

5. Xie W, Du L (2011) Diabetes is an inflammatory disease: evidence from traditional Chinese Medicines. Diabetes Obes Metab 13: 289-301.

6. Falchetti A, Masi L, Brandia ML (2011) Thiazolidinediones and bone. Clin Cases Miner Bone Metab 4: 103-107.

7. García-Giménez JL, Sanchis-Gomar F, Pallardó FV (2011) Could thiazolidinediones increase the risk of heart failure in Friedreich's ataxia patients? Mov Disord 26: 769-771

8. vanWijk JP, de Koning EJ, Martens EP (2003) Thiazolidinediones and blood lipids in type 2 diabetes. Arterioscler Thromb Vasc Biol 23:1744-1749.

9. Rubins HB, Robins SJ, Collins D (2002) Diabetes, plasma insulin, and cardiovascular disease: subgroup analysis from the Department of Veterans Affairs high-density lipoprotein intervention trial (VA-HIT). Arch Intern Med 162: 2597-2604.

10. Cai G, Liu B, Liu W (2007) Buyang Huanwu Decoction can improve recovery of neurological function, reduce infarction volume, stimulate neura proliferation and modulate VEGF and Flk1 expressions in transient focal cerebral ischaemic rat brains. J Ethnopharmacol 113: 2: 292-299.

11. Lu ZM, Yu YR, Tang H (2005) The protective effects of Radix Astragali and Rhizoma Ligusticichuanxiong on endothelial dysfunction in type 2 diabetic patients with microalbuminuria. Sichuan Da Xue Xue Bao Yi Xue Ban 36 529-532.

12. Chan CM, Chan YW, Lau CH (2007) Influence of an anti-diabetic foot ulcer formula and its component herbs on tissue and systemic glucose homeostasis. J Ethnopharmacol109: 10-20.

13. Xu ME, Xiao SZ, Sun YH (2006) Effects of astragaloside IV on pathogenesis of metabolic syndrome in vitro. Acta Pharmacol Sin 27: 229-236.

14. Lau KM, Lai KK, Liu CL (2012) Synergistic interaction between Astragali Radix and Rehmanniae Radix in a Chinese herbal formula to promote diabetic wound healing. J Ethnopharmacol 14: 250-256.

15. Pradhan AD, Manson JE, Rifai N (2001) C-reactive protein, interleukin 6, and risk of developing type 2 diabetes mellitus. JAMA 286: 327-334.

16. Yin MJ, Yamamoto $Y$, Gaynor RB (1998) The anti-inflammatory agents aspirin and salicylate inhibit the activity of IKB kinase- $\beta$. Nature 396: C77-80.

17. Vozarova B, Weyer C, Lindsay RS (2002) High white blood cell count is associated with a worsening of insulin sensitivity and predicts the development of type 2 diabetes. Diabetes 51: 455-461.

18. Hoo RL, Wong JY, Qiao CF (2010) The effective fraction isolated from Radix Astragali alleviates glucose intolerance, insulin resistance and hypertriglyceridemia in $\mathrm{db} / \mathrm{db}$ diabetic mice through its anti-inflammatory activity. Nutr Metab (Lond) 7: 67.

19. Piconi L, Quagliaro L, Da Ros R (2004) Intermittent high glucose enhances ICAM-1, VCAM-1, E-selectin and interleukin-6 expression in human umbilical endothelial cells in culture: the role of poly (ADP-ribose) polymerase. $J$ Thromb Haemost 2: 1453-1459.

20. Shoelson SE, Lee J, Goldfine AB (2006) Inflammation and insulin resistance. J Clin Invest 116: 1793-1801.

21. Fortes PC, de Moraes TP, Mendes JG (2009) Insulin resistance and glucose homeostasis in peritoneal dialysis. Perit Dial Int 29: S145-S148.

22. Aguirre V, Uchida T, Yenush L (2000) The c-Jun NH2-terminal kinase promotes insulin resistance during association with insulin receptor substrate-1 and phosphorylation of Ser307. J Biol Chem 275: 9047-9054.

23. Hotamisligil GS, Peraldi P, Budavari A (1996) IRS-1-Mediated Inhibition of Insulin Receptor Tyrosine Kinase Activity in TNF-alpha -and Obesity-Induced Insulin Resistance. Science 271: 665-668.

24. King GL (2008) The role of inflammatory cytokines in diabetes and its complications. J Periodontol 79: 1527-1534.
25. Yin X, Zhang Y, Wu H (2004) Protective effects of Astragalussaponin I on early stage of diabetic nephropathy in rats. J Pharmacol Sci 95: 256-266.

26. Bastard JP, Jardel C, Bruckert E (2000) Elevated levels of interleukin 6 are reduced in serum and subcutaneous adipose tissue of obese women after weight loss. J Clin Endocrinol Metab 85: 3338-3342.

27. Bastard JP, Maachi M, van Nhieu JT (2002) Adipose tissue IL-6 content correlates with resistance to insulin activation of glucose uptake both in vivo and in vitro. J Clin Endocrinol Metab 87: 2084-2089.

28. Hotamisligil GS, Shargill NS, Spiegelman BM (1993) Adipose expression of tumor necrosis factor-alpha: direct role in obesity-linked insulin resistance. Science 259: 87-91.

29. Frøkiær H, Henningsen L, Metzdorff SB (2012) Astragalus Root and Elderberry Fruit Extracts Enhance the IFN- $\beta$ Stimulatory Effects of Lactobacillus acidophilus in Murine-Derived Dendritic Cells. PloS One 7: e47878.

30. Li M, Yu L, She T (2012) Astragaloside IV attenuates toll-like receptor 4 expression via NF-kB pathway under high glucose condition in mesenchymal stem cells. Eur J Pharmacol 696: 203-209.

31. Evans JL, Goldfine ID, Maddux BA (2002) Oxidative stress and stressactivated signaling pathways: a unifying hypothesis of type 2 diabetes. Endocr Rev 23: 599-622.

32. Xu Y, Feng L, Wang S (2011) Phytoestrogen calycosin-7-O- $\beta-D-$ glucopyranoside ameliorates advanced glycation end products-induced HUVEC damage. J Cell Biochem 112: 2953-2965.

33. Xu Y, Feng L, Wang S (2011) Calycosin protects HUVECs from advanced glycation end products-induced macrophage infiltration. $J$ ethnopharmacol 137: 359-370.

34. Giacco F, Brownlee M (2010) Oxidative stress and diabetic complications. Circ Res 107: 1058-1070.

35. Baynes, JW (1991) Role of oxidative stress in development of complications in diabetes. Diabetes 4: 405-412.

36. Ramasamy R, Vannucci SJ, Yan SS (2005) Advanced glycation end products and RAGE: a common thread in aging, diabetes, neurodegeneration, and inflammation. Glycobiology 7: 16R-28R.

37. Lal MA, Körner A, Matsuo Y (2000) Combined antioxidant and COMT inhibitor treatment reverses renal abnormalities in diabetic rats. Diabetes 49 : 1381-1389.

38. Studer RK, Craven PA, DeRubertis FR (1997) Antioxidant inhibition of protein kinase $\mathrm{C}$-signaled increases in transforming growth factor-beta in mesangial cells. Metabolism 46: 918-925.

39. Hsieh HM, Wu WM, Hu ML (2011) Genistein attenuates d-galactose-induced oxidative damage through decreased reactive oxygen species and NF-KB binding activity in neuronal PC12 cells. Life Sci 88: 82-88.

40. Fan Y, Wu DZ, Gong YQ (2003) Effects of calycosin on the impairment of barrier function induced by hypoxia in human umbilical vein endothelial cells. Eur J Pharmacol 481: 33-40.

41. Yu D, Duan Y, Bao Y (2005) Isoflavonoids from Astragalusmongholicus protect PC12 cells from toxicity induced by L-glutamate. J Ethnopharmacol 98: 89-94.

42. Wright E, Scism-Bacon JL, Glass LC (2006) Oxidative stress in type 2 diabetes: the role of fasting and postprandial glycaemia. Int J Clin Pract 60: 308-314.

43. Yki-Järvinen H (2004) Thiazolidinediones. N Engl J Med 351: 1106-1118.

44. Kavak S, Ayaz L, Emre M (2012) Effects of rosiglitazone with insulin combination therapy on oxidative stress and lipid profile in left ventricular muscles of diabetic rats. Exp Diabetes Res: 905683.

45. Spigoni V, Picconi A, Cito M (2012) Pioglitazone improves in vitro viability and function of endothelial progenitor cells from individuals with impaired glucose tolerance. PloS One 7: e48283. 
46. Shen P, Liu MH, Ng TY (2006) Differential effects of isoflavones, from Astragalusmembranaceus and Puerariathomsonii, on the activation of PPARa, PPARy, and adipocyte differentiation in vitro. J Nutr 136: 899-905.

47. Gao, D, Guo, Y, Li, X (2013) An Aqueous Extract of Radix Astragali, Angelica sinensis, and Panaxnotoginseng Is Effective in Preventing Diabetic
Retinopathy. Evid Based Complement Alternat Med 2013: 578165.

48. Motomura, K, Fujiwara, Y, Kiyota, N (2009) Astragalosides isolated from the root of astragalus radix inhibit the formation of advanced glycation end products. J Agric Food Chem 17: 7666-7672. 